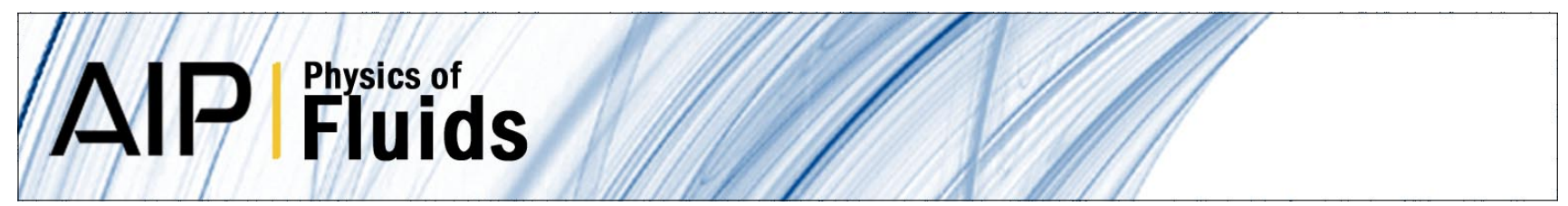

\title{
A plane mixing layer with crossshear
}

P. Atsavapranee and M. Gharib

Citation: Phys. Fluids 6, 2880 (1994); doi: 10.1063/1.868114

View online: http://dx.doi.org/10.1063/1.868114

View Table of Contents: http://pof.aip.org/resource/1/PHFLE6/v6/i9

Published by the AIP Publishing LLC.

\section{Additional information on Phys. Fluids}

Journal Homepage: http://pof.aip.org/

Journal Information: http://pof.aip.org/about/about_the_journal

Top downloads: http://pof.aip.org/features/most_downloaded

Information for Authors: http://pof.aip.org/authors

\section{ADVERTISEMENT}

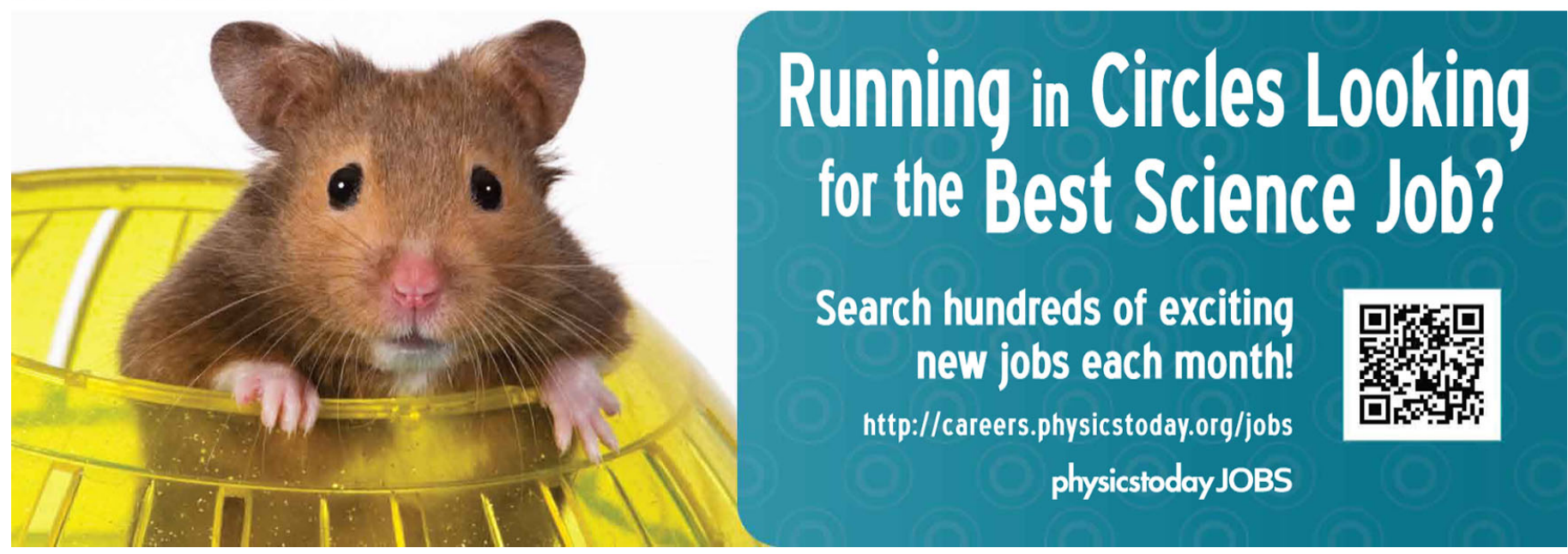




\title{
A plane mixing layer with cross-shear
}

\author{
P. Atsavapranee and M. Gharib \\ Graduate Aeronautical Laboratories, California Institute of Technology, Pasadena, California 91125
}

(Received 2 February 1994; accepted 13 May 1994)

The effect of cross-shear on a nominally two-dimensional plane mixing layer is investigated. A temporal mixing layer is generated with a stratified tilting tank similar to that used by Thorpe [ $\mathrm{J}$. Fluid Mech. 32, 693 (1968)], and cross-shear is introduced to this mixing layer to simulate situations the authors believe are widespread in industrial and natural flows. A new type of "co-rotating" streamwise vortices arises from the introduction of cross-shear, and the mixing rate is found to be greatly enhanced.

Since Bernal and Roshko ${ }^{1}$ reported on the discovery of streamwise vortical structures in a nominally plane mixing layer, studies of this type of "counter-rotating" streamwise vortices have occupied the efforts of many researchers in the field. These efforts, both numerical and experimental, involve attempts to force counter-rotating streamwise vortices by various means: spanwise numerical perturbations (Corcos and $\operatorname{Lin}^{2}$ ), corrugated splitter plates (Lasheras and $\mathrm{Choi}^{3}$ ), strip heaters (Nygaard and Glezer ${ }^{4}$ ), etc. However, most natural shear flows and many industrial flows involve complexities of different kinds, the most important in our opinion being the transient nature of the mean shear, the freedom of the mean shear to change in magnitude and direction with time. The nature of three-dimensionality in the mixing layers that involve this kind of complexity would, therefore, be inherently different from the scenario of the counter-rotating streamwise vortices. Some examples of relevance are swirling jet flows ${ }^{5}$ and shear flows in the ocean and atmosphere that are not constrained by sidewalls, nor restricted to only one direction of shear. A likely scenario in a complex atmospheric mixing layer, for example, would be that while a two-dimensional mixing layer is developing, cross-wind could blow over such a mixing layer introducing a crosscomponent of shear to that mixing layer. Another way to describe this same scenario is that the mean shear of the mixing layer changes amplitude and direction midway through its development. It is not hard to see then that some attention should be given to studying mixing layers that are complicated by the presence of cross shear.

In the attempt to investigate the effect of introducing cross-shear to a plane mixing layer, it is desirable to keep the geometry of the flow as simple as possible in order to avoid any unknown and undesirable effect. To this end, we find as ideal for our purpose an apparatus used extensively by Thorpe $^{6}$ in his study of mixing layers. In his 1968 paper, Thorpe $^{6}$ describes a very simple and elegant way that a temporally developing stratified mixing layer can be generated without the presence of a splitter plate, using an enclosed horizontal tank filled with a layer of salt water and a layer of fresh water of equal depths. The tank is tilted off the horizon generating the potentials for the two layers to accelerate in opposite directions, resulting in a velocity shear at the interface. The nature of the apparatus, in the absence of a splitter plate, allows a very "clean" development of the mixing layer, which is also largely unaffected by the end walls, at least for a short period of time. A similar tilting tank is constructed for the purpose of this study, but with the allowance for the tank to be rotated around a secondary axis. The tank, 5 in. $\times 30$ in. $\times 96$ in. in dimension, is first tilted around the main axis; and a certain time later, while the resulting shear layer is still developing, we tilt the tank around the secondary axis, generating the cross-shear. The apparatus was designed in a way so that the degree and the timing of the secondary tilt, relative to the main one, can be easily controlled with a computer. A schematic of the flow generated is represented in Fig. 1. The two axes of rotation are along the $z$ axis for the main shear and the $x$ axis for the cross-shear. The streamwise direction is defined as the direction of the main base flow ( $x$ axis), and the spanwise direction is along the axis of the main Kelvin-Helmholtz rollers ( $z$ axis).

Two types of flow visualization experiments have been carried out: one using the laser-induced fluorescence (LIF) technique to visualize two-dimensional planes and the other using a technique, similar to that used by Lasheras and Choi, ${ }^{3}$ to visualize the interface between the two streams by premixing the bottom layer with a high concentration of a fluorescent dye and illuminating from above with flash lamps to create an opaque interface. The specific gravities of the top and bottom layers are 0.998 and 1.085 , respectively, giving a density stratification of about $8 \%$, normalized by the average density of the two layers. The Reynolds number ranges from around 1500 at the appearance of the first Kelvin-Helmholtz structures to around 10000 after turbulent transition. The Richardson number of the main base flow, at the onset of Kelvin-Helmholtz instabilities is kept low in order to minimize buoyancy effects. Cross-shear of varying degrees were used, from $10 \%$ of the main shear to around $50 \%$. For the cases presented, the second tilt is initiated near the time of the onset of the main KelvinHelmholtz instabilities. It should be noted that if the cross shear is introduced while the main flow is still basically a flat two-dimensional vortex sheet, the resulting flow is no different than a normal two-dimensional shear layer, besides for the fact that the flow would be redirected along an oblique axis. But since the cross shear is introduced while the main Kelvin-Helmholtz structures are rolling up, the resulting flow is complex and three dimensional. The ensuing flow develops very fast, usually lasting only a few seconds before turbulent transition. The length scales used in the Reynolds number and the Richardson number are the maximum-slope 


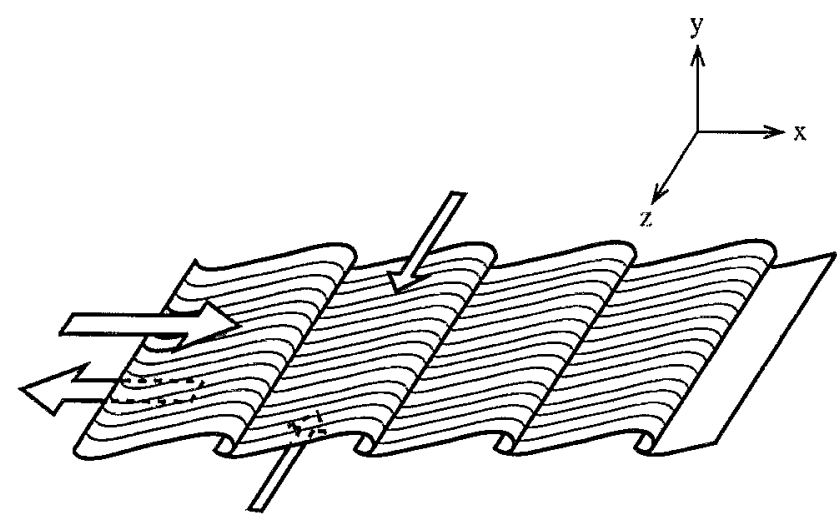

FIG. 1. A schematic showing the overall picture of a mixing layer with cross-shear.

thicknesses obtained from density profiles measured before and after each run using a conductivity probe. The Reynolds number and the Richardson number are defined as the following: $\mathrm{Re}_{i}=\Delta U \delta_{\rho} / \nu, \mathrm{Ri}_{i}=g \Delta \rho \delta_{\rho} / \rho_{\mathrm{av}}(\Delta U)^{2}$.

Figure 2 illustrates the top view of a mixing layer to which cross-shear is introduced. In these photographs only a

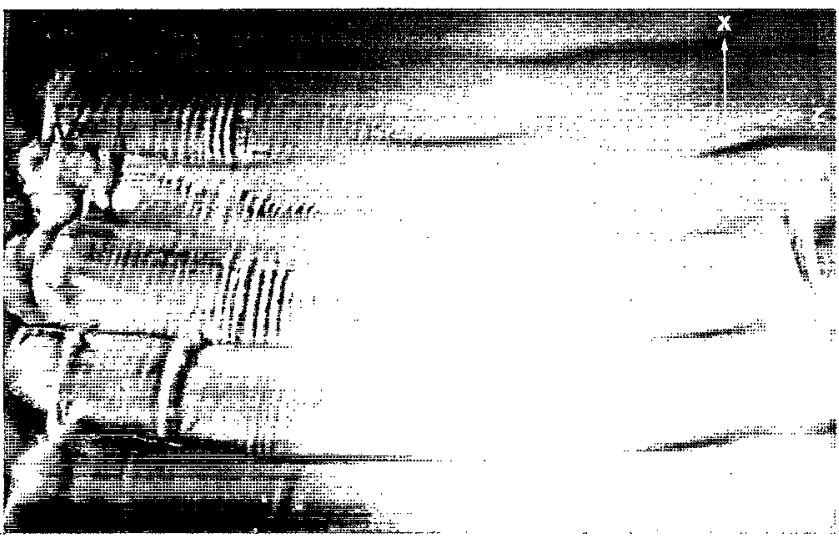

(a)

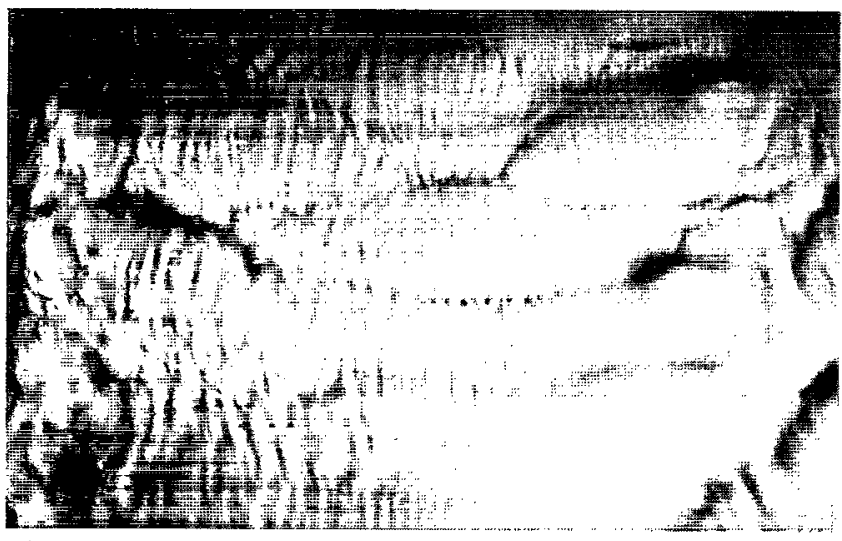

(b)

FIG. 2. Top view of a mixing layer to which a weak cross-shear is imposed. Streamwise streaks develop in the braid and increase in scale as they evolve. The times of (a) and (b) are 5.1 and $5.5 \mathrm{~s}$ after initiation of the main tilt. Cross-shear is introduced at $5 \mathrm{~s}$, shortly after the onset of the main KelvinHelmholtz instabilities (at $3.9 \mathrm{~s}$ ). The Reynolds number of the main shear is 2800. Richardson number is 0.03 for the main shear and 0.5 for the crossshear. The cross-shear is about $20 \%$ of the main shear.

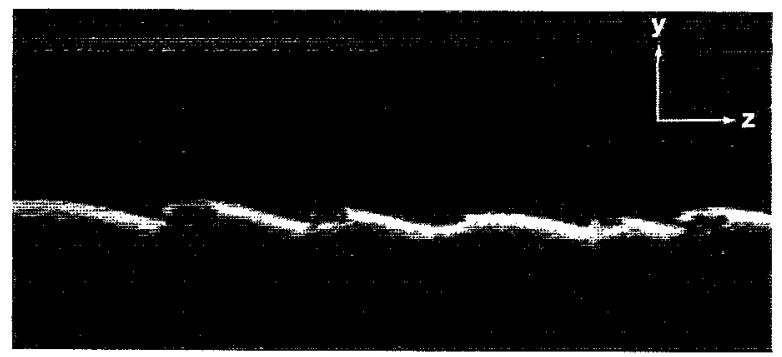

FIG. 3. A cross-sectional view of the streamwise streaks showing the "corotating" streamwise vortices. The image is taken $5.6 \mathrm{~s}$ after the initiation of the main tilt. Cross-shear is introduced at $4 \mathrm{~s}$, about the same time as the onset of the main Kelvin-Helmholtz instabilitities (at $4.1 \mathrm{~s}$ ). The Reynolds number of the main shear is 1600 . The Richardson number is 0.07 for the main shear and 0.15 for the cross shear. The cross-shear is approximately $50 \%$ of the main shear.

thin layer on the interface of the top and bottom layer is visualized. The dark lines represent the edges of vortical structures that form shadows when the interface is illuminated from top. The main shear gives a vorticity component in the negative $z$ direction, and the cross-shear in the negative $x$ direction. As expected, the usual Kelvin-Helmholtz instabilities develop as a result of the main shear, with the main rollers aligned along the $z$ axis (spanwise direction). In addition, streamwise streaks, which are roughly perpendicular to the main rollers, are observed to develop in the braid region [Fig. 2(a)]. The length scale of these streaks, after they first appear, is initially about 0.3 in., an order of magnitude lower than that of the main rollers, which have a wavelength of about 3 in. As the mixing layer develops, the length scale of the streamwise streaks increases, seemingly going through successive doublings in scale [Fig. 2(b)]. Eventually, the mixing layer goes through turbulent transition, and it is observed that transition occur earlier for a mixing layer with cross-shear than for the case without cross-shear.

The $y-z$ view or a cross-sectional view of these streamwise streaks illuminated by a laser sheet (Fig. 3) reveals that they seem to be structures of concentrated vorticity and that they are of the same sign everywhere. Qualitatively, the streamwise vortices behave very much like KelvinHelmholtz rollers in that they roll up and pair. Figure 4 is a sequence of pictures showing the pairing of two streamwise vortices. It is normal for these streamwise vortices to pair twice or three times, eventually making their sizes comparable to those of the main rollers, which pair much slower. Simple dimensional analysis shows that the streamwise vortices, when first appeared, have roughly the same length scale (normalized with the local braid thickness) and pairing time scale (normalized with the overturning time scale $\lambda / \Delta U)$ as normal Kelvin - Helmholtz rollers would.

It is clear from these observations that the streamwise streaks are, in fact, concentrated streamwise vortical structures, and that they are direct consequences of the crossshear. Deep understanding of the nature of these streamwise vortices is still a long way away, but a rudimentary picture of the dynamics can be derived from the clues available. The 


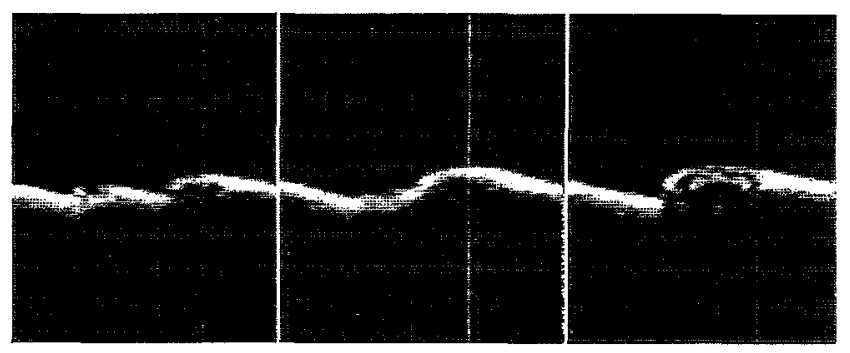

FIG. 4. A sequence of pictures showing two "co-rotating" streamwise vortices in the process of pairing. The conditions are the same as in Fig. 3. The times of the sequence are $5.60,5.73$, and $5.85 \mathrm{~s}$ after the initiation of the main tilt.

introduction of cross-shear to a developing plane mixing layer essentially has the effect of imposing uniform streamwise vorticity everywhere in the mixing layer. This streamwise vortex sheet feels the presence of the flow field of the main mixing layer, and as a result becomes unstable, giving rise to the concentrated streamwise vortices. The stability problem of the streamwise vortices can be qualitatively explained. In the braid region of the main rollers around the stagnation point, the local flow field looks like that of Hiemenz flow. This flow field creates a strain field that would magnify by stretching any streamwise vorticity present. With a uniform streamwise vortex sheet imposed upon the mixing layer, the stretching action in the braid region would magnify the streamwise vorticity there and make it very likely for the sheet to go unstable in a KelvinHelmholtz-like manner. The braid flow field would further stretch these Kelvin-Helmholtz-like streamwise vortices, which would go through pairings similar to normal KelvinHelmholtz rollers. The fact that the streamwise vortices are so perpendicular to the spanwise ones is most likely, due to the fact that the main Kelvin-Helmholtz rollup depletes the braid of the spanwise vorticity and concentrates most of it in the rollers. The vorticity in the braid, after the introduction of cross-shear, is therefore mainly in the streamwise direction. The length scale of the streamwise vortices would scale on the thickness of the layer in the braid, where they originate. Since the braid is continually being stretched thinner and thinner, it is expected and, in fact, observed that the length scale of the streamwise vortices would be lower than that of the main rollers, and would depend on how thick the braid is when the cross-shear is introduced.

It is important to point out that the streamwise vortices appear, even though the cross-shear is very weak (the Richardson number for the cross-shear $=0.5$ ), implying that the rollup of the streamwise vortex sheet into concentrated vortices are intimately related to the strain field imposed by the main Kelvin-Helmholtz rollers. In fact, if the streamwise vortex sheet is present alone, it would not roll up, since the Richardson number is well above the critical number of 0.25 . Therefore, one cannot think of the flow as two separate and independent arrays of perpendicular Kelvin-Helmholtz rollers superimposed upon each other. Rather, the streamwise vortices are a result of a secondary instability in the braid region of the main Kelvin-Helmholtz rollers.

The problem of a mixing layer with cross-shear has a strikingly strong parallel to the stability analysis of Lin and Corcos. ${ }^{7}$ In Sec. IV of that work, the authors make a case for modeling the development of a uniform streamwise vortex sheet in the braid region of a two-dimensional shear layer. They approximate the local flow field around the stagnation point of the shear layer with a plane strain field and then numerically study the dynamics of the streamwise vortex sheet under that strain field.

The observed streamwise vortices also have a close parallel in a helically perturbed nominally axisymmetric jet. Martin and Meiburg ${ }^{5}$ has observed Kelvin-Helmholtz-like streamwise vortices of the same sign in the braid regions of a numerical helical jet. Figure 1 shows the overall view of a mixing layer with cross shear. The streamwise vortices appear as streamwise streaks when viewed from top. These streamwise vortices are different from the counter-rotating type, as described by Bernal and Roshko.' Counter-rotating streamwise vortices would reveal "mushroom" shapes in the cross-sectional plane, and the wavelength would not increase because the mushroom structures do not pair.

In conclusion, we believe the co-rotating streamwise vortices play a significant role in determining the nature of the turbulent transport in the mixing layer. Not only would the characteristics of the turbulence be different, but also the degree of mixing would be greater as well. Measurements of the mixing layer thickness, before and after the tank tilting, show that mixing layers with cross-shear get 1.6-2.0 times as thick as the mixing layers without cross-shear. In a simple two-dimensional mixing layer, it is the main rollers that do most of the entrainment and mixing, with the braid contributing little to the process. The presence of these streamwise vortices in the braid would greatly enhance mixing, not only by entraining and mixing fluid themselves, but also by interacting with the main rollers, creating more small-scale turbulence. Since the streamwise vortices grow to a size comparable to that of the main rollers, the interaction between the two perpendicular structures can be expected to be significant, resulting in more mixing and earlier transition.

${ }^{1} \mathrm{I}$. P. Bernal and A. Roshko, "Streamwise vortex structures in plane mixing layers," J. Fluid Mech. 170, 499 (1986).

${ }^{2}$ G. M. Corcos and S. J. Lin, "The mixing layer: Deterministic models of a turbulent How. Part 2. The origin of the three-dimensional motion," J. Fluid Mech. 139, 67 (1984).

"J. C. Lasheras and H. Choi, "Three-dimensional instability of a plane free shear layer: An experimental study of the formation and evolution of streamwise vortices," J. Fluid Mech. 189, 53 (1988).

${ }^{4}$ K. J. Nygaard and A. Glezer, "Evolution of streamwise vortices and generation of small-scale motion in a plane mixing layer," J. Fluid Mech. 23I, 257 (1991).

${ }^{5}$ J. E. Martin and E. Meiburg, "The three-dimensional evolution of axisymmetric jets perturbed by helical waves," in $8 t h$ Symposium on Turbulent Shear Flows, Technical University of Munich, 1991, p. 6-3.

${ }^{6} \mathrm{~S}$. A. Thorpe, "A method of producing a shear flow in a stratified fluid," J. Fluid Mech. 32, 693 (1968).

S. J. Lin and G. M. Corcos, "The mixing layer: Deterministic models of a turbulent flow. Part 3. The effect of plane strain on the dynamics of

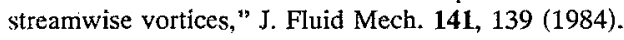

